\title{
Review: geriatric health services after discharge do not improve mental state in elderly people
}

Cole MG. The impact of geriatric post-discharge services on mental state. Age Ageing 2001 Sep;30:415-8.

\author{
QUESTION: In elderly people, do geriatric health services after discharge improve \\ mental state?
}

\section{Data sources}

Studies were identified by searching Medline (1975 to May 2000), HealthSTAR (1975 to May 2000), and the Cochrane Database of Systematic Reviews (2000, issue 1). Bibliographies of relevant articles were also searched for additional references.

\section{Study selection}

Studies published in English or French were selected if they were original research, randomised or nonrandomised controlled trials of a geriatric post discharge service, and $\geqslant 1$ measure of mental state was reported.

\section{Data extraction}

Data were extracted on country, study design, sample size, subject selection, key components of the intervention, duration of follow up, quality of the study, and outcomes. Outcomes included depression, morale, life satisfaction, contentment, emotional function, social activities, and self perceived health or cognition.

\section{Main results}

11 randomised controlled trials (3814 patients, $\geqslant 60 \mathrm{y}$ of age in 9 trials) met the selection criteria. Interventions included patient assessment and follow up by a multidisciplinary team (physician, nurse, physiotherapist, occupational therapist, social worker or support workers) ( 5 trials), patient assessment and follow up by a nurse ( 3 trials), help provided by a home aide or attendant ( 2 trials), and visitation by a volunteer ( 1 trial). Small improvements in morale, contentment, emotional function, life satisfaction, and self perceived health were reported in 3 trials. The intervention and control groups did not differ for any of the outcomes in 8 trials.

\section{Conclusion}

In elderly people, geriatric health services after discharge do not improve mental state.

\section{COMMENTARY}

An impaired mental state can adversely affect the patient's ability to function, and also affects whether a caregiver can look after the patient. This review by Cole has examined the mental state (ie, cognition, depression, and anxiety) of geriatric patients after discharge from medical units. Of 98 potential references found through searching computer databases, 11 included some measure of mental state upon follow up after discharge. Little impact on mental state at post discharge follow up was noted from the varying outcome measures used in the 11 trials. The need for further and more extensive research in this area was suggested.

Depression and cognitive impairment have been found to be main predictors of morbidity and mortality in a number of medical conditions, particularly in the elderly. Death due to cardiac disease or stroke occurs more often when depression is present. ${ }^{12}$ Cognitive impairment is a common sequela of coronary artery bypass surgery. ${ }^{3}$

This study suggests the need for increased attention to mental state measures in outcome research of hospitalised geriatric patients both during hospitalisation and at post discharge visits.

$$
\begin{array}{r}
\text { Irene E Ortiz, MSW, MD } \\
\text { University of New Mexico } \\
\text { and Veterans Administration Medical Center } \\
\text { Albuquerque, New Mexico, USA }
\end{array}
$$

1 House A, Knapp P, Bamford J, et al. Mortality at 12 and 24 months after stroke may be associated with depressive symptoms at 1 month. Stroke 2001;32:696-701.

2 Penninx BW, Beekman AT, Honig A, et al. Depression and cardiac mortality: results from a community-based longitudinal study. Arch Gen Psychiatry 2001;58:221-7.

3 Newman MF, Kirchner JL, Phillips-Bute B, et al. Longitudinal assessment of neurocognitive function after coronary-artery bypass surgery. N Engl J Med 200 1;344:395-402.
Source of funding: not stated.

For correspondence: Dr M G Cole, St Mary's Hospital Center, Montreal, Quebec, Canada. martin.cole@ ssss.gouv.qc.ca 